\title{
LA FENOMENOLOGÍA COMO DINÁMICA DE LA MANIFESTACIÓN ${ }^{1}$
}

\author{
Prof. Dr. Renaud Barbaras \\ Université Paris I, Francia \\ Institut universitaire de France
}

\begin{abstract}
Resumen: El análisis patočkiano del aparecer es solicitado por tres exigencias convergentes: la manifestación no pertenece al orden de lo manifestado, no es obra de un sujeto, y no obstante reviste la posibilidad de un aparecer subjetivo. Este artículo pretende mostrar cómo la consideración rigurosa de las coordenadas del aparecer conduce en línea recta hacia una teoría dinámica de la manifestación, que ve en el movimiento, irreductible sin lugar a duda a un simple desplazamiento, aquello que constituye el sentido último de ser del mundo como del sujeto, permitiendo así articularlos entre sí.
\end{abstract}

Descriptores: Patočka $\cdot$ Mundo $\cdot$ Aparecer $\cdot$ Manifestación.

Abstract: The Patočkian analysis of appearing is governed by three convergent requirements: manifestation does not belong to the realm of that which is manifested, it is not the work of a subjectivity and, nevertheless, it involves the possibility of subjective appearing. This paper aims at showing how, by taking into account rigorously these dimensions of appearing, we are led straight to a dynamic theory of manifestation, that understands movement, obviously irreducible to a mere displacement, as that which constitutes the ultimate sense of being of the world as the subject, and thus enables us to articulate them.

Keywords: Patočka $\cdot$ World $\cdot$ Appearing $\cdot$ Manifestation.

La fenomenología asubjetiva de Patočka evidentemente no tiene el sentido de un esfuerzo por marginar la subjetividad, no es una fenomenología sin sujeto. Por el contrario, ella se inscribe, de manera asumida, en el marco del a priori universal de correlación entre el ente trascendente y sus modos subjetivos de donación, $a$ priori por el cual Husserl circunscribió el espacio de la fenomenología. La ambición de Patočka es la de recuperar este a priori en su verdadero contenido, lo que a la vez supone tanto especificar el sentido de ser auténtico de los entes en correlación como

\footnotetext{
${ }^{1}$ E-mail: renaudbarbaras@orange.fr. Este texto ha sido traducido por Manfredi Moreno y fue primero publicado bajo el título "La phénoménologie comme dynamique de la manifestation" in Les Etudes philosophiques, (C) Presses Universitaires de France/Humensis, 2011. Agradecemos la editorial por autorizar esta traducción.
} 
revelar lo que los vincula más allá de la diferencia en su modo de ser, en resumen, caracterizar la relación en su ser. Por lo tanto, la fenomenología asubjetiva sólo se opone al subjetivismo husserliano, en la medida en que este subjetivismo demuestra que Husserl es infiel con sus propios principios y sus propias exigencias. Como sabemos, este subjetivismo se manifiesta en la asimilación de la epojé a una simple reducción a la región conciencia, reducción que no es ella misma sino el revés de la constitución del mundo en la conciencia sobre la base de sus vivencias inmanentes (hyle y nóesis). En otras palabras, el aparecer mismo, cuya función es hacer ver los entes que aparecen, retirándose a su favor, se encuentra subordinado a un tipo singular de apareciente, a saber, las vivencias inmanentes dadas adecuadamente a la conciencia, es decir por sí mismas. Este aparecer que, en cuanto pura ostensión de entes, no es otra cosa que estos entes mismos en su ser manifiesto, se encuentra relacionado con un ente singular, la conciencia, que acaba asumiendo por sí sola toda la carga de este aparecer. La subjetividad, que no puede sino significar la fenomenalidad misma en la medida en que nuestra existencia se refleja en ella, se vuelve entonces el predicado de un ente singular que tiene el privilegio de acceder a sí mismo en transparencia. Desde la perspectiva de Patočka, este enfoque muestra una falta de radicalidad y da testimonio de una forma de ingenuidad, es decir, de una dependencia ante la actitud natural en su sentido más profundo, cuestión que se le escapa naturalmente a Husserl. En efecto, la actitud natural es la tentativa de dar cuenta del aparecer a partir de un apareciente, presuponiéndolo así secretamente cuando intentamos comprender la esencia. La posición ingenua de la existencia del mundo y la tentativa de dar cuenta de la aparición misma del mundo a partir de esta existencia es un gesto que pertenece típicamente a esta ingenuidad y a esta circularidad, ya que equivale a tratar de dar cuenta del aparecer sobre la base de lo que él hace aparecer del lado trascendente. Sin embargo, el hecho de que Husserl se apoye en este ente privilegiado que es la vivencia, aunque dado adecuadamente a él mismo, no cambia nada: "Hay un campo fenomenal, un ser del fenómeno como tal, que no puede reducirse a ningún ente que aparezca en su seno y que, por lo tanto, es imposible explicar a partir del ente, sea este de especie naturalmente objetivo o egológicamente subjetivo" (Patočka, 1988, p. 239).

Puesto que el sujeto aparece y que la vivencia se manifiesta a sí misma, el sujeto procede de una ley general del aparecer y es por lo tanto imposible, a menos que se caiga en la circularidad, recurrir a él para dar cuenta de este aparecer como tal. El descubrimiento fundamental de la fenomenología es el de la independencia radical del aparecer en su legalidad frente al apareciente y sus propias leyes: "La legalidad o, si se quiere, la estructura del aparecer es totalmente independiente de la estructura de las cosas ónticas; no se puede deducir la manifestación como tal ni de las estructuras objetivas ni de las estructuras psíquicas" (Patočka, 1983, p. 39). La 
única pregunta entonces en conformidad con la autonomía radical del aparecer, con su irreductibilidad a todo apareciente, es la siguiente: ¿cuál es el significado del aparecer en cuanto que en él se pueden dar tanto realidades trascendentes como realidades "subjetivas", "vivencias"? O, mejor dicho, situándose en el marco del a priori de correlación: ¿cómo pensar la aparición en su aparecer en la medida en que ella es conjuntamente aparición de un sujeto cuya existencia es apodíctica y de un mundo cuya existencia, presupuesta por toda aparición singular, es igualmente apodíctica? El sentido de la epojé patočkiana es la de allanar el terreno de una respuesta, procurando liberar las leyes del aparecer de cualquier dependencia con respecto a unos aparecientes y, en particular, a unas vivencias inmanentes. De esto se sigue que el ego, cuya apodicticidad implicaba, en Husserl, su exención de la neutralización va a ser de ahora en adelante incluido en el paréntesis de la epojé: “¿No será que la inmediatez de la donación del ego sea un prejuicio, que la experiencia de sí tenga, al igual que la experiencia de las cosas, un a priori específico que vuelva posible el aparecer del ego?" (Patočka, 1988, p. 257). Este a priori pasa a ser el mundo mismo que, de ser una propiedad a priori del ego, tal como es caracterizado por Husserl, se vuelve un a priori del ego mismo Esto equivale a decir que toda aparición, incluso la del ego mismo, presupone la del mundo, que toda aparición implica por lo tanto la coaparición del mundo. Por eso la legalidad del aparecer envuelve, a título de su primer momento, y esto de manera no-contradictoria, la donación de este inmenso apareciente que es el mundo: "Consideramos como perteneciente a la estructura del aparecer en cuanto tal esta totalidad universal del apareciente, el gran todo, así como aquello a quien el ente aparece, la subjetividad (que tiene una estructura pronominal vacía, no identificable con un sujeto singular cerrado)" (Patočka, 1995, p. 177). Tal formulación equivale a restituir la correlación fenomenológica en su verdad, lo que excluye la versión subjetivista, es decir, como una verdadera codependencia de un mundo, totalidad intotalizable de entes, y de un sujeto (vacío pronominal).

Sin embargo, la cuestión del sentido de ser verdadero de este mundo y de este sujeto en el marco de la correlación repensada sigue abierta. Ahora bien, esta cuestión es inseparable de la del estatuto verdadero del aparecer como tal, es decir de la relación en ella misma, en la medida en que el sentido de ser del sujeto y del mundo remite en última instancia a la naturaleza de aquello que los articula entre sí. Quisiéramos mostrar cómo la consideración rigurosa de las coordenadas del aparecer conduce directamente a una teoría dinámica de la manifestación, que ve precisamente en el movimiento, cuyo significado queda por supuesto por determinar, lo que constituye el sentido de ser último tanto del mundo como del sujeto, y que permite, por consiguiente, articularlos entre sí.

Tratemos, por tanto, de aclarar el sentido de la manifestación a la luz de esta epojé radicalizada. En primer lugar, la autonomía de la manifestación significa 
que ella es independiente de todo apareciente, que ella no es de ningún modo tributaria de lo manifestado. Ahora bien, lo propio de lo que se manifiesta en la manifestación es que su modo de ser es el de la cosa o, más precisamente, el del ente: "La manifestación misma no es ninguna de estas cosas que se muestran. No es ni una cosa psíquica ni una cosa física, que se extiende en el espacio. Y, sin embargo, es la manifestación de estas cosas, tanto de las cosas que se extienden en el espacio como de las realidades tales como yo y mi pensamiento." (Patočka, 1983, p. 30). Esto equivale a decir que, puesto que ella es la condición de las cosas aparecientes, la manifestación remite a un modo de ser ajeno al de la cosa o al del ente. Por otra parte, de lo anterior se desprende que, si el sujeto es por cierto el destinatario del aparecer o de la manifestación, no es de ninguna manera la causa ni incluso la condición: él es lo que recibe la manifestación en lugar de ser lo que la hace o la constituye. Esto es lo que subraya claramente Patočka: "Yo pienso por tanto que la inversión la más apropiada debe consistir en el hecho que la subjetividad misma debe mostrarse como algo apareciente, como siendo parte de una estructura más profunda, como una cierta posibilidad esbozada e indicada en esta estructura a título de una de sus partes componentes. Esto no significa que sea posible un aparecer que no aparezca a nadie. Sin embargo, este alguien o algo no es ni el creador ni el portador. Lo que porta es la estructura, y aquello o aquel a quien aparece lo que aparece (el ente), es un momento y una parte integrante de esta estructura fundamental." (Patočka, 1983, p. 50). Efectivamente, decir que la subjetividad es un apareciente es reconocer que ella forma parte de un aparecer, o más bien de una estructura de aparecer de la que es un momento, una parte integrante. Esto significa dos cosas: por una parte, el aparecer no es portado por la subjetividad puesto que es este quien, por el contrario, la hace posible como surgimiento de este ente singular apareciente que es la vivencia; y por otra, el aparecer comprende la referencia a la subjetividad como una dimensión que le es constitutiva, de modo que la subjetividad es, sin lugar a duda, "parte integrante" de la estructura. Esto significa que el aparecer no es esencialmente subjetivo, en el sentido en que reposaría en la subjetividad, sino que es de una estructura tal que puede dar lugar al aparecer subjetivo, que puede comprender la posibilidad de la subjetividad. El sujeto viene, por lo tanto, a recibir y realizar una manifestación que es autónoma y, por así decirlo, más profunda que él.

Un breve análisis de la manifestación pone así de manifiesto tres exigencias convergentes: (1) la manifestación no pertenece al orden de lo manifestado no es una cosa; (2) no reposa en el sujeto, no es su obra; (3) sin embargo, comprende la posibilidad de una manifestación subjetiva, de un aparecer a. Por supuesto, la segunda condición solicita la primera en la medida en que la subjetividad implica por cierto la presencia de algo, de esta categoría de entes denominados vivencias. No es necesario decir que, al formular la situación de esta manera, entramos en el ámbito 
de la ontología puesto que el ente no puede ser referido por entero a la subjetividad trascendental. Se trata, por el contrario, de pensar una presencia o una venida del ente a la manifestación que no dependa de su aprehensión por la parte de un sujeto, en el entendido que esta aprehensión es ella misma tributaria del acontecimiento de la manifestación. Las coordenadas del problema determinan la solución. Puesto que la manifestación no puede ser referida al orden óntico ni realizada por este ente singular que es la conciencia, es necesario pensarla como una negación de lo óntico sin ser por ello un no-ser, esto es como un devenir. Si la manifestación no es una nada, sin ser por ello alguna cosa, no puede ser comprendida sino como el advenir mismo de alguna cosa. Dicho de otra manera, la manifestación debe ser pensada de manera dinámica, como un cierto proceso o un cierto movimiento, por el cual el ente precisamente se desvela o se descubre, en resumen, deviene él mismo. Cuando decimos que las cosas se manifiestan, ya no debemos entenderlo en el sentido que estarían dadas a una conciencia, que su ser cambiaría radicalmente en ser percibido o conocido, pero sí en el sentido que ellas son el sujeto de su manifestación, el artífice de su propio aparecer. Más precisamente, dado que las cosas no se vuelven como tales, es decir no se realizan en tanto cosas si no es solamente en y por la manifestación (decir que hay cosas y decir que aparecen, es decir lo mismo), debe reconocerse entonces que ellas son la obra de un movimiento de manifestación cuyo sujeto no es del orden de la cosa sino de un Fondo que no puede ser otro que el mundo mismo. Así, la afirmación según la cual el mundo es el primer momento de la legalidad del aparecer, es decir lo que aparece en toda aparición, debe entenderse ahora en modo dinámico: si el mundo aparece en toda aparición, es exactamente en la medida en que es lo que efectúa la aparición, es lo que constituye la fuente de esta. Así, esta forma del aparecer que es el mundo ${ }^{2}$ es al mismo tiempo el contenido más profundo puesto que no es otra cosa que lo que produce la aparición. La aparición es aquello cuyo artífice es el mundo. Sin embargo, es preciso añadir inmediatamente, para no proyectar la figura del ente sobre el mundo mismo, que este mundo no es nada de positivo fuera de esta obra misma, que no es otra cosa sino aquello de lo que es la fuente, un fondo que se confunde con lo que funda.

Un pensamiento riguroso sobre la manifestación requiere, por tanto, una cierta transformación metafísica. En efecto, podría ser fácil mostrar que la primacía de la vivencia y de la reflexión, característico del subjetivismo husserliano, remite en última instancia al predominio de una metafísica del ente y de la esencia, para la cual solo es verdaderamente lo que escapa al devenir, aquello que permanece inmutable. El marco aquí es platónico y, en el fondo, eleático. Si queremos entonces pensar el aparecer en su autonomía, se hace necesario pasar del plano del ser al

\footnotetext{
2 "Así la forma-de-mundo (Weltform) de la experiencia es a la vez lo que hace posible una experiencia de mundo" (Patočka, 1995, p. 214).
} 
del devenir, del plano de la esencia al del acontecimiento, del plano del ente al del movimiento. En algunos fragmentos valiosos, Patočka asume esta posición. Así, escribe en una breve nota: "Concepto de movimiento como fundamento movimiento concebido, no como movimiento del objeto, sino como obra de la physis antes de cualquier objetivación o subjetivación - la physis como esencia que es acontecimiento, esencia que adviene" (Patočka, 1995, p. 269). A la luz de las coordenadas de la manifestación, parece ser que el movimiento no es un simple desplazamiento ni incluso algo que, adviniendo a un objeto (cambio), presupondría su existencia, sino más bien el fundamento mismo. La esencia no reposa en ella misma y ya no constituye más el sentido último del ser: se sume en el devenir, es un acontecimiento o más bien su propio advenimiento. Ya no tenemos un movimiento que remitiría al ente como su sustrato, sino un acontecimiento en el que y por el que adviene, al mismo tiempo que su esencia, el ente mismo. Sin embargo, huelga decir que este movimiento, que es más profundo que el ente, es indiferente a la repartición de lo subjetivo y de lo objetivo, en la medida en que ambos son predicados del ente y, en consecuencia, lo presuponen. De esto se desprende también que, en cuanto fundamento, este movimiento permitirá dar cuenta de la distinción, en el seno del ente, entre lo objetivo y lo subjetivo: será un movimiento a la vez de objetivación y de subjetivación. Así, lo que hay, o más bien lo que es verdaderamente, no es más del orden del ser inmutable o del eidos: "Lo que está constantemente presente no es solamente el eidos, la forma, sino, del mismo modo y de manera aún más fundamental, la progresión continua del no-ser hacia el ser, la metabolé, el movimiento" (Patočka, 1988 a p. 133). Este movimiento-fundamento, que no es aún el movimiento de las cosas sino el proceso mismo de la physis como advenimiento de la esencia, es lo que podríamos llamar un movimiento de mundificación, en el sentido de que es por él que sobrevienen los entes que componen un mundo. El mundo es a la vez la fuente o la potencia (mundo mundificante) y su resultado, en forma de una multiplicidad fluyente de entes inacabados (mundo mundificado). Este movimiento del aparecer, Patočka lo nombra al menos una vez "protomovimiento" y lo describe así: "debe haber algo así como un movimiento por el cual el meollo del mundo constituya su contenido contingente y del cual el espacio-tiempo-calidad en totalidad es un sedimento." (Patočka, 1995, p. 157). Este texto extraordinario explicita la physis como acontecimiento de la esencia. El meollo del mundo, que no es otra cosa que la physis en tanto tal o el mundo mundificante, pasaje del no-ser hacia el ser, constituye la multiplicidad de entes intramundanos como contenido contingente, es decir, como el rostro finito y provisorio del pasaje, rostro que no deja de cambiar puesto que el pasaje mismo es continuo, la eclosión sin término de la physis. Esta figura del mundo (mundo mundificado) aparece como el contenido, o más bien el producto de sí mismo como proceso mundificante. De esto debemos 
concluir en primer lugar, lo que no deja de ser importante, que los movimientos en el seno del mundo, movimientos ónticos y ya no movimiento del aparecer, siguen siendo modalidades del protomovimiento en la medida en que participan del proceso de desaparición de los entes y del surgimiento de nuevos entes, en breve del proceso de mundificación. Por otra parte, el meollo del mundo sigue estando presente en el seno de los entes mundanos; algo de su profundidad, de su unidad y de su indivisibilidad primera perdura a nivel del mundo constituido. De hecho, el meollo del mundo se sedimenta al interior de lo múltiple en forma de un principio de unidad, de aquello que puede unirlos, a saber, el espacio, el tiempo y la calidad. Estas formas son la huella de la unidad originaria del mundo procesual al interior de la diversidad que ella misma genera, el testimonio de la potencia y de la indivisibilidad de la fuente en lo que ella misma produce. En este sentido, habría que distinguir tres sentidos del mundo: el mundo como protomovimiento, el mundo como contenido, es decir pluralidad depositada por este movimiento y, finalmente, el mundo como forma o principio de unidad (espacio-tiempo), sedimento del protomovimiento en su resultado, del fundamento en lo contingente.

Aparecer ya no significa darse a una conciencia o ser aprehendido por ella, es decir captado en su sentido o su ser conocido, sino salir del fondo, "fuera de la simple clausura que equivale a una noche obscura y, en este sentido, al no-ser" (Patočka, 1988ª , p. 100), llegar a la luz. La intuición fundamental es que las cosas no se nos darían, no se nos aparecerían si no se prestaran antes por ellas mismas a esta captación, si no vinieran por así decirlo al encuentro de esta aprehensión saliendo del retiro. En consecuencia, cabe preguntarse qué significa exactamente esta salida fuera del fondo que sabemos que procede de un movimiento, o más bien que ella define el sentido originario del movimiento. Dando por sentado que esta manifestación primera equivale a tomar lugar en el mundo, queda aún por determinar el sentido de este lugar. Es aquí donde es necesario tomar en consideración la tercera condición enunciada en un comienzo. La manifestación comprende esencialmente la posibilidad de una aparición subjetiva, de un aparecer a; ella comporta un horizonte subjetivo, incluso si no es del orden de la subjetividad, la manifestación es propiamente un dirigirse a un sujeto. Por tanto, es necesario partir de la aparición propiamente subjetiva para comprender lo que es el proceso originario de manifestación, ya que está por así decirlo codeterminado por el horizonte de la aparición subjetiva. Ahora bien, independiente del contexto teórico en el cual nos situamos, debemos reconocer que el aparecer subjetivo implica una dimensión constitutiva de identidad y, por tanto, de unidad. Como lo dice Pradines, "percibir es percibir algo", a saber, una realidad distinta de lo que la rodea y que requiere, por ello mismo, un principio de unidad. Es este principio de unidad, exigencia mínima del sentido, que nos permite separarla de su tejido material - por el cual mantiene una continuidad ontológica con su entorno y 
con el mundo- y de llevarla al plano del sentido, en breve, de conocerla. El aparecer propiamente tal señala el movimiento por el cual una realidad llega a ser su sentido, a su ser conocido: la conciencia no es otra cosa que una Bewusstsein. Es preciso concluir, a la luz de esta precesión con la que hemos caracterizado la manifestación, que nunca podremos llevar a cabo esta captación de la unidad, esta aprehensión de la cosa como tal, si esta no se hubiera de alguna manera unificado ella misma previamente, si la unidad no se hubiera ya precedido en la cosa. La determinación no es lo que el sujeto aporta, sino más bien lo que presupone.

Toda la dificultad es así la de comprender lo que puede significar una determinación que no se base en una aprehensión o un conocimiento, resumiendo, en una modalidad de ser ya subjetiva. Por supuesto, se podría replicar que un realismo de corte platónico se fundamenta precisamente en la idea que existe una realidad del eidos, una autonomía de la determinación. Pero, pensándolo bien, esta posición se encuentra siempre sustentada secretamente por la asimilación previa del ser por el ser-conocido $\mathrm{y}$, en este sentido, por una subjetivación, tan secreta como profunda, de la realidad. Establecer una realidad de la esencia, supuestamente independiente del cognoscente, equivale a separarla de su verdadero origen y proyectar así en el ser de la esencia el ser de la cosa mundana. En resumen, la esencia no es nunca una cosa sino solo el ser-conocido de la cosa, y hacer de ella una realidad autónoma significa separarla del fondo subjetivo sobre el que reposa. ¿Qué puede entonces significar la presencia de una determinación en el ámbito de una realidad que es independiente del sujeto? ¿Cuál puede ser su modo de ser habida cuenta de que no puede ser el de una cosa? Solo hay una respuesta posible, que viene a confirmar mediante la vía del aparecer subjetivo lo que había sido obtenido gracias a las dos primeras exigencias puestas en evidencia: no hay determinación sino mediante la referencia a un movimiento, como a lo que ese movimiento viene a realizar. La determinación no es una realidad cósica sino lo que un movimiento efectúa o logra y que, por ese motivo, rige su curso. En otros términos, el ser del sentido es un ser producido y, por lo tanto, su realidad no puede distinguirse del proceso del que solo es la norma inmanente. Esto es extremadamente importante porque de esto se desprende que esta determinación que el movimiento realiza, o más bien que no es sino como realización, no es por sí misma ni subjetiva ni objetiva. Ella es rigurosamente neutra, convirtiéndose en objetiva si se realiza como cosa y en subjetiva si se realiza como significación. Para ser más precisos, es la determinación misma que se realiza como objeto y como significación. Ahora bien, en la medida en que el ser de la determinación es el del movimiento - ella es lo que rige un proceso pero que no tiene como realidad otra cosa sino el proceso mismo -, se debe concluir que la diferencia entre lo objetivo y lo subjetivo, entre la determinación como cosa y la determinación como significado propiamente tal, se referirá a una distinción entre movimientos o entre modalidades de movimiento. La consecuencia 
de todo esto es que la subjetividad misma deberá ser comprendida como movimiento, correspondiendo a un cierto tipo de movimiento. La singularidad de la significación como tal no puede sino remitir a la singularidad del movimiento en el que se genera: la singularidad misma de este movimiento constituirá la especificidad del modo de existencia subjetivo. Comprender el real sentido de ser de la subjetividad equivale a dar cuenta del tipo de movimiento que ella es en cuanto que dicho movimiento hace ser la significación en su estado puro, produce de la determinación fuera de lo determinado - a diferencia de lo que sucede con los movimientos no subjetivos (no existenciales) que, por su parte, solo hacen advenir la determinación en lo determinado.

Así, decir que la unidad que es constitutiva del aparecer subjetivo no puede sino precederse en la realidad misma, es reconocer que la manifestación es en el fondo movimiento, única modalidad de ser conveniente para esta unidad. Solo hay unidad unificada y nunca unificante. En este sentido, la determinación de la manifestación como movimiento es confirmada desde el punto de vista de lo que constituye, en cierto modo, su punto de culminación. No obstante, por otra parte, reconsiderando la manifestación desde el punto de vista de su culminación subjetiva, nos estamos dando los medios para caracterizar con mayor precisión el movimiento que ella es: de acuerdo con su estatuto de dirigirse o de destino subjetivo, este movimiento no puede ser sino el de la realización de una unidad, de la constitución de una determinación. Si solo hay unidad unificada, entonces solo hay movimiento unificador. De esta manera, este movimiento de salida fuera del fondo por el cual Patočka viene a caracterizar la manifestación en su modo de ser más originario, debe ser comprendido como un proceso de individuación. Es en la medida en que el proceso de mundificación es un proceso de individuación, y en el que la salida fuera del fondo es el acceso a una forma de unidad, que se hace posible el aparecer subjetivo propiamente tal como aprehensión del objeto según su principio de unidad, es decir, la síntesis. Como lo escribe ejemplarmente Patočka: "Sentido de la identificación: no hay identidad porque sintetizo, pero sintetizo porque pongo el dedo en una identidad - el cambio, el proceso, la transformación son en ellos mismos unas identificaciones, son unas síntesis materiales, y mi síntesis subjetiva de identificación es simplemente la captación y el reconocimiento de esta identidad singular, de esta conexión al interior de las cosas" (Patočka, 1995, 32).

Es hora de notar que esta primera síntesis material no se efectúa sobre el fondo de un mundo ya constituido. Por el contrario, el mundo solo se constituye conjuntamente con esta individuación como la unidad de la multiplicidad de la que ella procede. En otras palabras, la unidad cerrada de los individuos mundanos y la unidad abierta del mundo nacen juntas de este primer proceso de individuación. Este Uno que es el ente apareciente adviene siempre en el seno de una unidad totalitaria que no es otra cosa que el mundo y que no se constituye, como unidad de esta 
multiplicidad, sino gracias a las individuaciones que advienen en su seno: la unidad del mundo (totalidad) y la de los entes mundanos (individualidad) se constituye y se determina conjuntamente. Como lo escribe Patočka: "Cada cosa adquiere su figura -se delimita- deviene un individuo al diferenciarse del resto. Este devenir traza fronteras frente a otra cosa, es un proceso de definición, de puesta en forma. Esta definición se hace respecto a todas las demás cosas, todas ellas determinadas. Este definir - el aparecer de la cosa como su ser. La cosa no solo "en ella misma", sino en todas las demás, ella es su propia relación de determinación con la totalidad de todo lo que es" (Patočka, 1995, p. 114). De ahí proviene una determinación del mundo como adjuntamiento, es decir como aquello que solo puede unificarse sobre el fondo de una multiplicación y cuya unidad es, en consecuencia, sinónimo de una diferenciación: el mundo no unifica sino separando. ${ }^{3}$ Por lo tanto, habría que decir que el mundo es aquello en lo que los entes difieren, en el doble sentido de aquello en lo que difieren y de aquello por lo que difieren.

Este análisis requiere varias observaciones. En primer lugar, como hemos señalado, la diferencia entre lo subjetivo y lo objetivo aparece definitivamente como subordinada: ambos proceden de la unidad, es decir de la síntesis, ambos remiten al movimiento, único modo de ser que conviene a la síntesis. Entonces, la diferencia entre lo subjetivo y lo objetivo comprende rigurosamente una diferencia entre movimientos: uno por el cual la unidad es realizada en la cosa o como cosa, el otro por el cual esta unidad es constituida por o como ella misma. Todavía falta precisar que, incluso en el caso del movimiento subjetivo (movimiento del cual procede la subjetividad), la unidad sigue siendo una unidad unificada, es decir una síntesis efectuada en la realidad. En la medida en que la unidad nunca puede realizarse fuera de aquello de lo que es la unidad, debe concluirse que ya no tiene sentido hablar de una realidad propiamente psíquica, subjetiva o mental. El aparecer subjetivo sigue siendo un aparecer, es decir algo que adviene a la cosa misma, una modalidad de la cosa apareciente, y su ser-para, que lo especifica como subjetivo, solo puede remitir al tipo de movimiento que se relaciona con ella. Así como el aparecer originario, condición del aparecer subjetivo, es el ser producido por el protomovimiento del mundo, el ser subjetivo es, por su parte, el ser producido por otro tipo de movimiento, que llamaremos movimiento de la existencia. La existencia será, por consiguiente, no un movimiento comparable a otros movimientos mundanos (aun si se efectúa necesariamente también de esa manera), sino este movimiento específico, comparable al proto-movimiento y situado en su prolongación, por la que el ente mundano se revela tal cual es. En cualquier caso, esto significa que el ver que es

\footnotetext{
${ }^{3}$ «Indiviso, él es quien a la vez separa y vincula todo; él es un todo no compuesto de partes, que es inconmensurable a cada parte, siendo al mismo tiempo contenido por completo en cada una" (Patočka, $1988^{\text {a }}$, p. 100).
} 
la percepción aparece como indisociable de un hacer, que la teoría es por entero praxis, o más bien que el aparecer solicita una dimensión más profunda que esta separación. La aparición del ente es indisociable de la acción que el sujeto ejerce sobre el ente, y evocar un movimiento de la existencia es reconocer que la donación del ente al sujeto solo es concebible como acción de un sujeto sobre el ente, al igual que la aparición primera y anónima del ente era indisociable de un protomovimiento de individuación.

En segundo lugar, si la determinación solo tiene una realidad dinámica, si su ser es inseparable de un producir, y, por otra parte, en la medida en que ningún resultado puede abolir el movimiento (el reposo es un momento del movimiento y no su negación), entonces es necesario concluir que la individualidad nunca es verdaderamente realizada. Esta individualidad solo existe como su propia promesa o su propio porvenir: en los términos de Gilbert Simondon, ella comporta una dimensión o una carga de preindividualidad irreductible. Si el ser de la unidad es su haber devenido, solo hay unidad presuntiva, en el horizonte, y su vocación es de permanecer irrealizable. Entonces podríamos leer el pasaje hacia el aparecer subjetivo, es decir la dimensión dirigida de la fenomenalidad, en términos de un progreso hacia la individuación, de una etapa necesaria en el proceso regido por una unidad cuya realización no se encuentra sino por venir. Desde luego, en la medida en que no es posible afirmar la más mínima teleología, es preciso atenerse al registro del "como si", empero podemos afirmar que todo sucede como si la unidad realizada en y por el protomovimiento del mundo solicitara un movimiento de otra naturaleza permitiéndole separarse de la masa insuficientemente diferenciada del mundo, para así realizarse como la unidad que ella es. Sea como sea, la unidad es al movimiento en general lo que la melodía o una composición musical es a cada uno de sus elementos: "cada elemento solo es una parte de algo que le excede, que no se encuentra allí de plano como una figura acabada, algo que más bien, preparado en todas las singularidades, permanece siempre, en un cierto sentido, por venir, todo el tiempo en que la composición se escucha" (Patočka, 1995, p. 108).

Esta concepción de la manifestación como movimiento se inscribe en el marco de una reflexión sobre Aristóteles, hasta el punto de que es en los textos dedicados a Aristóteles que podemos encontrar las afirmaciones más importantes de Patočka en torno a su concepción de la fenomenalidad. Esta referencia puede justificarse en dos niveles, bien articulados entre sí. En primer lugar, el movimiento tiene en Aristóteles una significación ontológica. En efecto, él no es un estado, sino un pasaje de la potencia al acto, es decir una realización de ser; así pues, es expresión a la vez de un defecto ontológico, constitutivo del mundo sublunar, y del acto de superarlo o de subsanarlo. Si el mundo sublunar se caracteriza por el movimiento, es porque la sustancia no puede acceder a la actualidad plena, y no lo puede porque 
este mundo es el de la materia, lugar de la indeterminación y de la potencia. De todas formas, el movimiento - habría que decir el cambio - porta el ente hacia aquello que él es, por supuesto en primer lugar en términos de la sustancia (genesis) pero también de la cantidad, de la calidad y del lugar. Por otra parte, Patočka interpreta a Aristóteles en el horizonte de una concepción unitaria de la fenomenalidad, que integra la subjetivación (el aparecer a), a título de una dimensión, por cierto constitutiva, de un proceso general de fenomenalización. En otras palabras, no se trata de afirmar, como lo hacemos a menudo, que la filosofía aristotélica de la naturaleza, dominada por el marco teleológico de la actualización de un sentido, de la realización, sería fruto de una simple proyección antropomórfica. Patočka muestra, en un texto decisivo, que es exactamente lo contrario y que no tiene entonces sentido hablar de antropomorfismo o de subjetivismo en lo que concierne la física y la metafísica aristotélicas. A propósito, una semejante interpretación encontraría una inevitable objeción, que la debilitaría al instante de manera considerable: si es posible que proyectemos estructuras humanas sobre fenómenos naturales, ¿no es porque algo en el modo de ser de esos mismos fenómenos nos incita a ello? Patočka, por su parte, interpreta la concepción aristotélica del movimiento en un sentido completamente distinto, haciendo eco a su propia concepción de la fenomenalidad: "Si el antropomorfismo significa un subjetivismo, la intención de Aristóteles es justamente la contraria. No se propone subjetivizar el mundo, de "animarlo", y de "espiritualizarlo". Al contrario, intenta descubrir estructuras asubjetivas que puedan englobar y también explicar, desde principios lo más universales, los fenómenos humanos, la comprehensión y el comportamiento humanos". Añade que por esta razón Aristóteles se encuentra en las antípodas del idealismo moderno en la medida en que no busca el sujeto en las cosas, como tampoco trata de introducirlo en ellas: "Él se esfuerza sin embargo por encontrar una medida común entre el mundo y el hombre, una concepción de la realidad del mundo que permita no dividirla en dos, al estilo de Platón y del cartesianismo, para así comprender al hombre como un caso particular, aunque fuera excepcional, de estructuras ontológicas generales" (Patočka, 1995, p. 30). No se trata, por lo tanto, de partir de la subjetividad y de su diferencia con el mundo para ver en la concepción aristotélica no solamente una proyección de su modo de ser en las cosas; al contrario, se trata de reconsiderar el ser del sujeto a partir de una modalidad de ser más general, en acorde con la totalidad del ente, y de la que la subjetividad solo es una especificación: es por cierto el hombre mismo quien debe ser comprendido en función de las estructuras del ser.

Entre las estructuras que tienden un puente entre lo humano y lo extrahumano, se encuentra precisamente, añade Patočka, el movimiento, lo que significa que nuestro movimiento debe ser él mismo comprendido como una modalidad de un movimiento más general que no es otro que el de la manifestación. En efecto, es 
a la luz de la concepción aristotélica que Patočka especifica su concepción de una manifestación originaria autónoma, asubjetiva, es decir anónima. Como es sabido, el ser no es, en Aristóteles, un género supremo, sino de entrada dividido según las categorías que, todas, remiten a la primera entre ellas, la sustancia, que aparece en consecuencia como el sentido verdadero del ser. En Aristóteles, el ser toma el rostro del ente individual. El movimiento puede entonces solo ser comprendido en este marco, como lo que conduce la sustancia individual a ella misma y, en consecuencia, la actualiza también según estas otras categorías que son la cantidad, la calidad y el lugar. Por último, cabe recordar que, si la sustancia puede ser comprendida en tres sentidos, es la quiddidad, es decir en el fondo la determinación (aquello que es el ente), que es la más originaria. En tanto que afecta la sustancialidad de la sustancia, el movimiento consiste necesariamente en un proceso de determinación, llevando así la sustancia a su quiddidad. Más precisamente, el movimiento no puede crear de la determinación, puesto que el acto precede siempre la potencia ("el hombre engendra al hombre"): se limita a actualizarla. Ello significa, en los términos de Patočka, que la obra propia del movimiento no es la de crear lo que sea, sino la de reunir las determinaciones, la de realizar su ser conjunto: "El movimiento reúne, vincula unas con otras las determinaciones de un mismo sustrato, (...) volviéndolas simultáneamente actuales. Así, el movimiento de maduración de una manzana ocasiona el encuentro, en un mismo sustrato, de la dulzura, el grosor, un color específico, un olor, etc." (Patočka, 1988ª p. 129). O sea que el movimiento sintetiza, es incluso exactamente esta síntesis material mencionada anteriormente, que precede y funda la síntesis subjetiva. Por supuesto, esta síntesis "opera" de acuerdo con la ley o la regla que caracteriza tal o cual ser: "Estas leyes y estas reglas son el sentido propio del ser: ellas determinan lo que puede o no puede ser simultáneamente actualizado en un mismo ser" (Patočka, 1988 a , p. 131). En efecto, puesto que todas las determinaciones están presentes en potencia en el mundo, la determinación de un ente solo puede significar una síntesis, un cierto ser conjunto. Hacer advenir tal ser (una manzana madura), es entonces hacer advenir las determinaciones cuya conjunción corresponde a este ser determinado (color, dulzura, etc.): es permitir un encuentro en conformidad a una regla. Ahora bien, en la medida en que el ser es fundamentalmente sustancia y en el que la sustancia significa ante todo la determinación, la quiddidad, el advenimiento de esta posee necesariamente una significación ontológica: significa el acceso de la sustancia al ser o más bien el advenimiento del ser como sustancia. Esto es lo que Patočka reconoce claramente: "Puesto que son las determinaciones del sustrato que clarificamos utilizando las palabras "es", "hay", de esto se sigue que es el movimiento lo que hace que las cosas sean lo que son - el movimiento es un factor ontológico fundamental" (Patočka, $1988^{\mathrm{a}}$, p. 129). 
Por lo tanto, encontramos inscrito en el marco aristotélico, la teoría dinámica de la manifestación que habíamos primeramente deducido de las coordenadas mismas de la fenomenalidad. Lo importante, para Patočka, es que, en este contexto, somos capaces de dar sentido a una individuación que no es estrictamente la obra de la subjetividad, a una determinación que es inseparable del movimiento que la hace advenir. Existe, por tanto, una articulación fundamental, que aflora en Aristóteles, entre una teoría ontológica del movimiento, que hace del movimiento un factor ontológico fundamental ("que hace que las cosas sean lo que son") y una metafisica de lo individual, para la cual el ser significa ante todo el ente individual. En efecto, el ser solo depende del movimiento en la medida en que requiere estar separado, desmarcado de otros, en breve, en la medida en que es esencialmente individual. Inversamente, el movimiento no tiene verdaderamente sentido sino como aquello que delimita, circunscribe, hace advenir lo individual. El movimiento es por esencia discriminatorio, separador: cambiar, es siempre avanzar hacia una nueva determinación, distinguiéndose a la vez de aquello que se era y de todo aquello que no. Como dice Patočka muy profundamente: "El movimiento es lo que hace aparecer que haya, por un tiempo determinado, un lugar en el mundo para una realidad singular determinada entre otras realidades singulares" (Patočka, 1988a , p. 103). Esto significa que el movimiento no crea nada sino que sintetiza y, sintetizando, distingue o separa. Delimitando una realidad singular, le da lugar y, con ello, la hace aparecer: no hay aparición sin separación, y no hay separación sin síntesis. El cambio es por cierto separador, razón por la cual la ontología del movimiento y la metafísica del individuo se solicitan mutuamente: si el ser es lo individual, es decir lo separado, solamente el movimiento como potencia separadora puede hacer ser. Esto es precisamente lo que Patočka descubre en Aristóteles: "La ontología de Aristóteles hace referencia al hecho que sin duda hay en el mundo individuos, es decir entes, que no se encuentran simplemente separados por nosotros del continuum de la experiencia, y que al contrario por esencia son una separación, un ordenamiento, un proceso de determinación, un despertar y un encaminarse desde el ser impropio al ser propio" (Patočka, 1995, p. 30). Como ya lo hemos visto con anterioridad, hay sin duda una unidad que precede y funda la síntesis subjetiva y que no puede sino tener el sentido de una síntesis material, de un hacer venir en conjunto, de una unificación que es al mismo tiempo una separación. Así lo subraya Patočka nuevamente: "La cosa, la ousia, lo que llamamos sustancia, se determina en cuanto tiene un límite, en cuanto se delimita de una cierta manera, y esta delimitación, este entrar en un límite, significa que es la cosa misma la que se está dibujando, la que se desmarca, la que se de-fine. ¿Qué es en el fondo la definición? La palabra misma nos da la respuesta: finis, un fin, una frontera, un límite" (Patočka, 1983, p. 170). La definición es en primer lugar una delimitación, delimitación que solo 
puede advenir en y por la obra separadora del movimiento; o más bien, en tanto que introduce un finis, una frontera, la delimitación que el movimiento permite es por cierto el sentido primero de la definición, es decir de la determinación. Es por lo que sin duda Patočka es particularmente sensible a esta fórmula aristotélica: "El movimiento es de alguna manera la vida de las cosas" (Patočka, 1988 a , 103, p. 129). Ella significa que el movimiento no es exterior a la cosa, sino que, todo lo contrario, el movimiento es a la cosa lo que la vida es al organismo, a saber, aquello que no solamente le pertenece, sino que lo hace ser y lo conserva en su ser manteniendo su separación respecto de los otros entes, oponiéndose cada vez al retorno a la fusión o a la indiferenciación con el entorno.

Por último, queda por comprender en qué sentido este proceso ontológico de individuación es un proceso de manifestación. Apoyándonos sobre lo que constituye de una cierta manera la culminación, hemos podido caracterizar el protomovimiento como proceso de individuación. Sin embargo, en la medida en que este protomovimiento es un movimiento de manifestación originaria, aún queda por explicar en qué sentido la individuación es fruto de la manifestación. El pasaje por Aristóteles nos permite precisamente comprender cuál sentido de la manifestación se encuentra implicado en la utilización de este término cuando hablamos del surgimiento del ente mundano antes de toda aprehensión subjetiva. Después de haber consignado que, en Aristóteles, la cosa como sustancia, que es en sí misma una forma (quiddidad), se define limitándose, él añade: "De esto se desprende que se trata de un carácter de la cosa misma, ¿pero de qué carácter? Es cuestión del carácter de su descubrimiento, de su manifestación" (Patočka, 1983, p. 170). El encadenamiento teórico es el siguiente: la delimitación producida en el movimiento es un descubrimiento y este descubrimiento es una manifestación en un sentido originario, o incluso: manifestarse es ser descubierto, pero ser descubierto es ser delimitado. En efecto, lo que así se descubre es el proceso por el cual algo que ya estaba allí no podía sin embargo aparecer, porque permanecía inaccesible a la visión, es decir disimulado. Ahora bien, existen dos maneras de estar disimulado, de las que resulta fácil mostrar que rigen dos direcciones incompatibles en el seno de la historia de la metafísica: por recubrimiento, en el sentido literal de la interposición o de la superposición de algo; por fusión o indistinción con el entorno. Es este segundo sentido de la disimulación que está en juego aquí. El movimiento descubre, no en el sentido de que retira lo que cubre, sino en el sentido de que permite un descubrimiento circunscribiendo y, por lo tanto, separando el ente de su medio. Así, la individuación es manifestación porque es descubierto o descubrimiento y es descubrimiento en cuanto es delimitación. Este descubrimiento ontológico es evidentemente la condición del descubrimiento perceptivo, o más bien, funciona como una "percepción" inmanente y anónima, percepción que va a venir a 
prolongar la percepción "subjetiva", en cuanto que, en el contexto de este primer descubrimiento, revela el contorno mismo, es decir la determinación, y capta el limite mismo, fuera de aquello que delimita.

Sin embargo, no podemos quedarnos en este estado de cosas si queremos pensar por entero el movimiento de la manifestación o la manifestación como movimiento. En efecto, es hora de recordar que el movimiento aristotélico, movimiento que es siempre de actualización, presupone un sustrato - lo que cambia - conduciendo de la privación (de determinación) a la posesión, es decir de un sustrato en el que la determinación no es actualizada a un sustrato en el que está plenamente presente. En este sentido, el movimiento presupone el ser en movimiento sin jamás producirlo: es la marca de un defecto de ser y sigue siendo, si se puede decir, un predicado ontológico derivado. En breve, se encuentra subordinado al marco previo de la sustancia y aparece como la manifestación de la distancia irreductible de la sustancia (como materia) respecto a sí misma (como forma). En tanto que está "privada de", el sustrato que se mueve es determinado negativamente, es una llamada a la determinación y el movimiento se limita entonces a hacerla venir, a conducir del sustrato a la quiddidad. Pero esta perspectiva no parece convenir a Patočka quien, otorgando al movimiento el estatuto mismo de acontecimiento de la esencia e identificándola de este modo con una physis, le otorga pues un estatuto mucho más fundamental, propiamente ontogenético. Por esto mismo Patočka reivindica una radicalización de la concepción aristotélica del movimiento. Esta radicalización no puede sino consistir en una concepción del movimiento según la cual este no adviene a un sustrato previo, sino que hace advenir el sustrato mismo y, con ello, la cosa en movimiento. El movimiento no es más lo que le sucede a alguna cosa sino aquello por lo que una cosa sucede: el movimiento hace realmente ser. Esto significa que deposita o unifica las determinaciones y, haciendo esto, hace ser el sustrato como sustrato de esas determinaciones. Entonces, no se debe hablar, en todo rigor, ni de la manzana madura ni del sustrato-manzana, sino de un movimiento de maduración o de madurez, movimiento que une determinaciones, que sintetiza y, por lo mismo, hace ser el sustrato en tanto que el lugar de esas determinaciones, lo sintetizado por esta síntesis. Lo que verdaderamente hay, no es ni el sustrato ni la determinación sino la venida o el proceso de esta, en la medida en que no hay otra determinación que la de su propio advenimiento: no el ser-maduro ni el sustrato-manzana sino la maduración misma. No hay ser maduro sino como movimiento de maduración, y es este movimiento quien produce conjuntamente la unidad de la determinación y la identidad del sustrato, o incluso el ser-conjunto y el lugar que así delimita. Es posible reanudar aquí la comparación aristotélica evocada anteriormente. Decir que el movimiento es la vida de las cosas, equivale a decir que es lo que las hace ser y que solo las puede mantener en el ser haciéndolas ser continuamente: es la 
identidad que reposa en la perennidad y no a la inversa. Esta identidad es dinámica, la determinación es producida y es por lo que no puede proceder de un sustrato previo; afirmar que la determinación es su propio producir, es reconocer que el sustrato proviene como aquello que ella viene a habitar. Es por lo que, en el marco de esta radicalización del aristotelismo, Patočka acaba por definir el movimiento como "vida original que no recibe su unidad del sustrato conservado, sino que crea ella misma su propia unidad y la de la cosa en movimiento. Solo el movimiento así concebido es movimiento original" (Patočka, 1988a , p. 103).

Es tanto como decir que el enfoque de Patočka consiste en comprender el movimiento ontogenético, es decir la pareja genesis-phtôra ${ }^{4}$ como entregando la esencia misma del movimiento. Este no presupone el ente constituido, sino que constituye el ente: es movimiento de la sustancia en el sentido de la emergencia y de la desaparición del ente. Así, en el contexto fenomenológico, la manifestación asubjetiva solo puede ser comprendida desde el movimiento solo bajo la condición de pensarlo como un movimiento ontogenético, movimiento por el cual el ente sale de la noche oscura, sinónimo del no-ser, y accede así al ser. Sin embargo, al igual que la consideración de la síntesis subjetiva conducía a una caracterización de la manifestación originaria como movimiento de individuación, asimismo, la consideración de nuestro movimiento, en su singularidad, en la medida en que es una modalidad del movimiento en general, permite confirmar y, por así decirlo, reforzar la radicalización del aristotelismo que desemboca en una caracterización del movimiento originario como movimiento ontogenético. Así lo escribe a su vez Patočka: "si, en lugar de este movimiento en algo, en lugar de posibilidades que serían la propiedad, el tener de un algo idéntico que se realiza en ellas, suponemos más bien que este algo es su posibilidad, que no hay nada en él antes de las posibilidades y subyacentes a estas, que no vive íntegramente sino que por la manera en la que él es en sus posibilidades - tendremos una radicalización del concepto aristotélico de movimiento" (Patočka, 1995, p. 107). La formulación, así como el contexto, indica que lo que Patočka tiene aquí en vista no es otra cosa que el movimiento de nuestra existencia y su planteamiento consiste en pasar del movimiento de nuestra existencia, en tanto que movimiento sin sustrato, a una esencia general del movimiento, de la que este movimiento es un testimonio eminente y que corresponde a la radicalización del concepto aristotélico. En efecto, Patočka saca aquí conclusiones de Heidegger: el Dasein tiene la característica de que existe, es decir que es su posibilidad, que es un poder-ser. Esto significa que la existencia del Dasein no es la realización de una posibilidad dada previamente, lo que sería retroceder al plano categorial, sino que es por el contrario el movimiento por el cual la posibilidad se descubre en y por su

\footnotetext{
${ }^{4}$ Nota traductor: conceptos griegos que remiten respectivamente al nacimiento (genesis) y a la muerte (phtôra), cf. Barbaras (2003), p. 101.
} 
propia realización. El Dasein es lo que es según el modo del tener que ser: su ser, su identidad, no precede el movimiento, sino que al contrario es lo que el movimiento realiza. En breve, el Dasein es realización en el doble sentido de un cumplimiento y de una venida a la realidad. Nos enfrentamos aquí a un movimiento muy singular ya que hace advenir al sujeto del que es el movimiento; es un movimiento que precede y funda su propio sujeto o su propio sustrato y lo hace ser determinándolo. El advenimiento de las determinaciones es al mismo tiempo el nacimiento de su sujeto: la constitución de lo determinado es relativo del surgimiento de su determinación, de suerte que al final la pertenencia misma de la determinación a lo determinado es realizada por este surgimiento. La existencia es una realización en la cual lo que se realiza solo surge en lo que la realiza. Ahora bien, si queremos inscribir esta idea de la existencia en una teoría general del movimiento en lugar de ver en ella un modo de ser absolutamente singular que solo podríamos proyectar sobre una naturaleza exterior, es necesario entonces admitir que la esencia del movimiento es realización en el sentido que venimos de precisar, a saber, constitución del sustrato, del ser en movimiento desde la determinación que este movimiento hace advenir. Es un movimiento que produce su propio sujeto, sujeto que, por lo mismo, no está nunca terminado, quedando siempre al horizonte de sí mismo. Así, la consideración de este singular modo de ser que es nuestra existencia nos lleva a reconocerla como inscrita en un proceso general de manifestación, en un movimiento ontogenético del que nuestra existencia no es sino una atestación privilegiada.

Así pues, es a condición de referir la manifestación al movimiento tal como lo hemos definido, a saber, como proceso por el cual el ente adviene en su individualidad, que seremos capaces de comprender cómo la subjetivación no funda la manifestación, sino que la acoge y la prolonga. No somos nosotros sino más bien el mundo que "hace" la manifestación (y el mundo no es en realidad otra cosa que ese hacer): una cosa solo nos aparece si primero ella aparece por así decirlo en y por ella misma, si ella es antes descubierta, lo que quiere decir circunscrita y por lo tanto individualizada. Es necesario por tanto distinguir dos sentidos, o más bien dos grados de la manifestación: la aprehensión subjetiva de una unidad supone de hecho un descubrimiento previo, la autoconstitución de una unidad que no puede ser sino la obra de un movimiento individuante. Patočka lo señala al menos una vez: "El desvelamiento presupone la determinabilidad; en este sentido, la delimitación es una condición. La delimitación y el desvelamiento pueden ser subsumidos bajo el concepto global de manifestación. El movimiento es el fundamento de toda manifestación" (Patočka, 1988a , p. 132). Esta breve frase resume el conjunto de la teoría patočkiana de la manifestación. Tenemos una primera manifestación que "no es manifestación para el sujeto, sino más bien manifestación como ingreso en la singularidad, devenir. Es una manifestación en la cual las cosas singularizadas 
son ellas mismas interiormente indiferentes, una manifestación que no es manifiesta a ella misma, una manifestación sumida en la oscuridad nocturna del ente y del ser originarios" (Patočka, 1988a , p. 100). De esta primera manifestación, hay que distinguir lo que Patočka denomina "la apertura secundariamente humana", (Patočka, 1988 a , p. 100) que no es más la apertura del ente por el ser según el modo dinámico que venimos de exponer, sino apertura del ente por el hombre. Esta manifestación corresponde a lo que comúnmente se entiende por aparecer: corresponde a la apertura subjetiva, a lo que Patočka denomina "desvelamiento" por oposición a delimitación.

Por supuesto, toda la dificultad es la de comprender esta manifestación segunda sin volver a introducir prejuicios subjetivistas, es decir sin salir del marco general de la teoría asubjetiva de la manifestación. Para ello, es necesario retomar el final del breve texto que venimos de citar: "El movimiento es el fundamento de toda manifestación". En efecto, preguntar en qué consiste el aparecer "subjetivo" es como preguntar qué es lo que singulariza nuestro movimiento en relación con los otros movimientos del proceso ontogenético general. Ahora bien, nuestro movimiento se caracteriza propiamente por encontrarse sujeto a una doble determinación. Por un lado, somos entes del mundo y, por ello, somos frutos del proceso general de individuación, somos "ingreso en la singularidad, devenir". Como lo escribe Patočka, "Nuestra propia individuación pertenecería también a este universo de la individuación primordial englobando todo lo que se revela inicialmente en su simple venida, en su despliegue, su nacimiento y su desaparición, sin participación interior al ser, sin interés para el ser" (Patočka, 1988 a , p. 100). Sin embargo, por otra parte, nuestro movimiento tiene la singularidad, por la cual se distingue de todos los otros, que su realización no carece de una relación al ser, envuelve precisamente una "participación interior al ser", un "interés por el ser". Esto equivale a decir que somos unos entes para los cuales les va en su ser este mismo ser, de suerte que nuestra propia realización es indisociable de un movimiento que no proviene más del ser, sino que va hacia él, que no es fruto de una producción del ente por el ente, sino más bien de un desvelamiento del ser por el ente que nosotros somos. Es por lo que Patočka puede continuar así: "La característica del movimiento que nos es especifica sería sin embargo la no-indiferencia al ser, el interés por el propio ser y, conjuntamente, por el ser del ente en general, sobre el fundamento de una nueva manera en la que el ser condiciona el ente - no simplemente en su emergencia y su desaparición, sino a su vez en tanto que claridad que vuelve posible el encuentro por dentro, al interior del universo" (Patočka, 1988a , p. 101). ${ }^{5}$ Lo propio de nuestro movimiento es que se realiza como interés por el ser y por su propio ser, interés que

\footnotetext{
${ }^{5}$ Ver también: Patočka (1983), p. 205.
} 
no es otra cosa que lo que Patočka denomina desvelamiento. Este desvelamiento es la claridad que vuelve posible el encuentro: el existente que somos capta el ente a la luz del ser.

Por lo tanto, la comprehensión de la singularidad de nuestra existencia reposa por entero en la comprehensión de la manera en que nos relacionamos con los otros entes, en la medida en que la realización de nuestra existencia pasa por el desvelamiento, la manifestación segunda de estos entes. El movimiento propio a la existencia, movimiento por el cual ella se individualiza, se confunde con aquel en y por el que ella lleva el ente al parecer, es decir prolonga la individuación mundana bajo la forma de una síntesis desveladora. Por consiguiente, si no queremos presuponer nada en cuanto a lo que se debe entender por subjetividad, cabe preguntarse en qué consiste el movimiento de la existencia. No se trata pues, como estaríamos tentados de hacerlo, de pensar nuestro movimiento desde el desvelamiento subrepticio comprendido como percepción o conocimiento sino, todo lo contrario, de comprender el desvelamiento desde la singularidad de nuestro movimiento. Todo converge por tanto hacia una interrogación sobre el movimiento de la existencia, que aparece por cierto como el centro de gravedad y, en consecuencia, la cuestión más difícil de la fenomenología de Patočka. En cuanto nuestra existencia participa de la manifestación, en cuanto la manifestación subjetiva se inscribe en el proceso general (asubjetivo) de la manifestación, nuestra existencia es movimiento. Con lo cual la singularidad de la manifestación subjetiva se apoya por entero en la especificidad de los movimientos de la existencia, el ser de la subjetividad remite por entero al modo de ser del movimiento que la sostiene. Entender lo que significa aparecer a un sujeto requiere entender lo que significa existir para un sujeto; entender lo que significa existir para un sujeto equivale a determinar lo que son sus movimientos; pero determinar lo que son sus movimientos equivale rigurosamente a comprender aquello que ellos hacen. 


\section{BIBLIOGRAFÍA ${ }^{6}$}

Barbaras, R. (2003). Vie et intentionnalité: recherches phénoménologiques. Paris: Vrin.

Patočka, J. (1983). Platon et l'Europe. Lagrasse: Verdier.

Patočka, J. (1988'). Le Monde naturel et le mouvement de l'existence humaine. Dordrecht, Boston, London: Kluwer Academic Publishers.

Patočka, J. (1988). Qu'est-ce que la phénoménologie ? Grenoble: Millon.

Patočka, J. (1995). Papiers phénoménologiques. Grenoble: Millon.

${ }^{6}$ Nota traductor: todas las traducciones son realizadas por el traductor refiriéndose a la obra publicada en francés. 